01

\title{
Форма поверхности вертикального жидкого моста между двумя параллельными твердыми плоскостями с учетом силы тяжести при малых числах Бонда
}

\author{
(C) Е.В. Галактионов, ${ }^{1}$ Н.Е. Галактионова, ${ }^{2}$ Э.А. Тропп ${ }^{1}$ \\ ${ }^{1}$ Физико-технический институт им. А.Ф. Иофре РАН, \\ 194021 Санкт-Петербург, Россия \\ ${ }^{2}$ Санкт-Петербургский политехнический университет Петра Великого, \\ 195251 Санкт-Петербург, Россия \\ e-mail: evgalakt@mail.ru
}

(Поступило в Редакцию 24 апреля 2017 г.)

Дана вариационная постановка задачи о форме боковой поверхности вертикального жидкого $\theta$-моста малого объема, находящегося между двумя параллельными твердыми плоскостями с учетом силы тяжести в осесимметричном случае. Построен алгоритм итерационного процесса решения задачи для малых чисел Бонда. Исследована зависимость числа решений от высоты жидкого моста.

DOI: $10.21883 /$ JTF.2017.10.44989.2297n

\section{Введение}

Результаты теоретического исследования профиля поверхности жидкой капли малого объема при наличии зоны трехфазного контакта необходимы для решения многих научных и технологических проблем [1,2]. В частности, для изучения формы жидких менисков, образующихся в процессе роста кристаллов по способу Степанова [3]. В этом случае жидкая капля расплава находится между двумя твердыми поверхностями с разными свойствами (например, между молибденовым формообразователем и кристаллической затравкой). Такая жидкая капля в разных источниках называется по-разному, так, в монографии Я.Е. Гегузина [4] еe называют „раздавленной каплей“, в иностранной литературе - „жидким мостом“ или „капиллярным мостом“ („liquid bridge“, „capillarity bridge“ ), в работе [5] „сплюснутой каплей“, в настоящей работе используется термин ,жидкий мост“.

В одной из первых работ, посвященных изучению жидких мостов [6], автор предлагает рассматривать два типа объектов, а именно мосты с фиксированным контуром контакта ( $r$-bridges) и с фиксированным углом смачивания ( $\theta$-bridges). Далее, следуя [7], можно выделить два основных направления исследования: изучение эволюции формы жидких мостов и изучение их устойчивости относительно различных возмущений. В литературе изучаются жидкие мосты между двумя параллельными твердыми плоскостями [6], между двумя сферами [8], между двумя осесимметричными твердыми телами [7].

При этом используются как асимптотические, так и численные методы решения уравнений. В работе [9] построена асимптотика формы поверхности горизонтального $\theta$-моста при малых числах Бонда. Библиография по рассматриваемой тематике представлена в работах $[7,9]$.
Настоящая работа посвящена нахождению формы поверхности вертикального жидкого $\theta$-моста между двумя твердыми плоскостями с учетом силы тяжести. Дана вариационная постановка задачи. Предложен алгоритм итерационного процесса нахождения приближенного решения задачи для случая малых чисел Бонда.

Исследование случая вертикального жидкого моста актуально для изучения процессов, имеющих место при затравлении кристаллов, выращиваемых из расплава [3].

\section{Вертикальный жидкий мост. Вариационная постановка задачи}

Рассмотрим случай жидкого моста, соприкасающегося с двумя плоскими твердыми поверхностями (дном и крышкой) (рис. 1). Жидкий мост в рассматриваемом случае представляет собой каплю жидкости малого объема, находящуюся между двумя параллельными твердыми плоскостями с заданными свойствами (сплюснутую каплю). Ввиду предполагаемой осевой симметрии будем решать задачу о нахождении профиля такой капли в цилиндрической системе координат $(r, z)$. Поверхностные натяжения между средами $\alpha_{13}, \alpha_{14}, \alpha_{34}, \alpha_{23}, \alpha_{24}$ соответственно. Область контакта капли с плоскостью $z=0$ (дном) - круг радиуса $r_{1}$, а область контакта капли с плоскостью $z=\hat{h}$ (крышкой) - круг радиуса $r_{2}$. Обозначим $u_{1}(r), u_{2}(r)$ - искомые функции, описывающие профили нижней $\left(u_{1}(r)\right)$ и верхней $\left(u_{2}(r)\right)$ частей капли. Область, разделяющая эти части („шейка“), - круг радиуса $r_{*}\left(r_{*} \geq 0\right)$. Так как нет никаких физических причин для появления заострения профиля капли, то в точке с абсциссой $r=r_{*}$ касательная к профилю капли должна быть вертикальной: $u_{1}^{\prime}\left(r_{*}\right)=-\infty, u_{2}^{\prime}\left(r_{*}\right)=+\infty$, $u_{1}\left(r_{*}\right)=u_{2}\left(r_{*}\right)$. Исключение составляет случай соприкосновения лежащей и висящей капель в одной точке. 


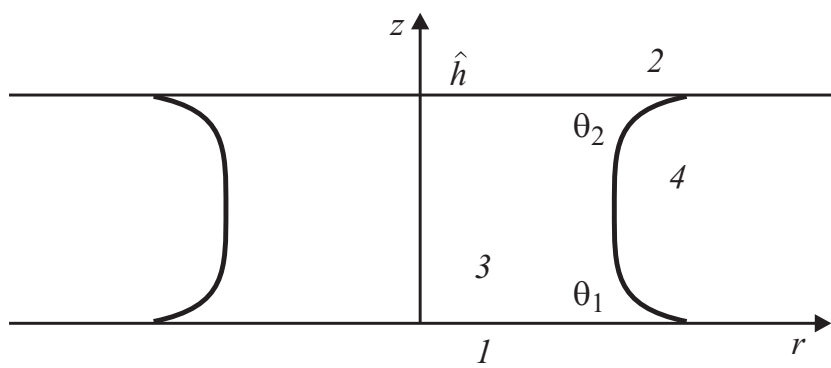

Рис. 1. Жидкий мост - в данном случае капля, находящаяся между двумя параллельными твердыми плоскостями. 1 и $2-$ твердые среды, 3 - жидкая среда, 4 - газообразная; $\theta_{1}, \theta_{2}-$ углы смачивания.

Предположим дополнительно, что углы смачивания $\theta_{1}, \theta_{2}$ не превосходят $90^{\circ}$. Тогда наша сплюснутая капля (жидкий мост) будет иметь катеноидальную форму $r_{*}<\min \left(r_{1}, r_{2}\right)$, а $u_{1}(r)$ и $u_{2}(r)$ будут однозначными функциями.

Объем капли считаем фиксированным:

$$
\begin{gathered}
I\left\{u_{1}(r), u_{2}(r)\right\}=2 \pi\left(\int_{r_{*}}^{r_{1}} u_{1}(r) r d r+\int_{r_{*}}^{r_{2}}\left(\hat{h}-u_{2}(r)\right) r d r\right) \\
+\pi\left(r_{*}\right)^{2} \hat{h}=V .
\end{gathered}
$$

Введем в рассмотрение функционал, включающий в себя поверхностную энергию и энергию силы тяжести. Поверхностная энергия, в свою очередь, состоит из части, соответствующей свободной поверхности капли, и части, соответствующей контакту жидкости с твердым телом. Исследуемый функционал можно записать в виде

$$
\begin{aligned}
& J\left\{u_{1}(r), u_{2}(r)\right\} \\
& =2 \pi \int_{r_{*}}^{r_{1}}\left\{\alpha_{34} \sqrt{1+\left(u_{1}^{\prime}\right)^{2}}+\frac{1}{2} g \rho u_{1}^{2}+\alpha_{13}-\alpha_{14}\right\} r d r \\
& +2 \pi \int_{r_{*}}^{r_{2}}\left\{\alpha_{34} \sqrt{1+\left(u_{2}^{\prime}\right)^{2}}-\frac{1}{2} g \rho\left(\hat{h}-u_{2}\right)^{2}+\alpha_{23}-\alpha_{24}\right\} r d r \\
& +\pi\left\{\alpha_{13}-\alpha_{14}+\alpha_{23}-\alpha_{24}+\frac{1}{2} g \rho \hat{h}^{2}\right\}\left(r_{*}\right)^{2},
\end{aligned}
$$

где $g$-ускорение силы тяжести, $\rho$-плотность жидкости.

Таким образом, получаем изопериметрическую задачу: найти минимум функционала (2) при условии, что функционал (1) принимает заданное значение $V$. В соответствии с теоремой Эйлера об изопериметрических задачах вводим расширенный функционал $(\lambda-$ множитель Лагранжа)

$$
\begin{aligned}
& J\left\{u_{1}(r), u_{2}(r)\right\}+\lambda I\left\{u_{1}(r), u_{2}(r)\right\} \\
& =2 \pi \int_{r_{*}}^{r_{1}} H_{1}\left(r, u_{1}(r), u_{1}^{\prime}(r)\right) d r+2 \pi \int_{r_{*}}^{r_{2}} H_{2}\left(r, u_{2}(r), u_{2}^{\prime}(r)\right) d r \\
& \quad+\pi\left\{\alpha_{13}-\alpha_{14}+\alpha_{23}-\alpha_{24}+\frac{1}{2} g \rho \hat{h}^{2}+\lambda \hat{h}\right\}\left(r_{*}\right)^{2},
\end{aligned}
$$

где

$$
\begin{gathered}
H_{1}\left(r, u_{1}(r), u_{1}^{\prime}(r)\right)=\left\{\alpha_{34} \sqrt{1+\left(u_{1}^{\prime}\right)^{2}}+\frac{1}{2} g \rho u_{1}^{2}\right. \\
\left.+\alpha_{13}-\alpha_{14}+\lambda u_{1}\right\} r, \\
H_{2}\left(r, u_{2}(r), u_{2}^{\prime}(r)\right)=\left\{\alpha_{34} \sqrt{1+\left(u_{2}^{\prime}\right)^{2}}-\frac{1}{2} g \rho\left(\hat{h}-u_{2}\right)^{2}\right. \\
\left.+\alpha_{23}-\alpha_{24}+\lambda\left(\hat{h}-u_{2}\right)\right\} r .
\end{gathered}
$$

Выполнив варьирование расширенного функционала, получим два уравнения Эйлера и два условия трансверсальности. Введем безразмерные переменные $\xi=$ $=r / V^{1 / 3}, w_{i}=u_{i} / V^{1 / 3}, i=1,2$ и безразмерные параметры $\mu=\lambda V^{1 / 3} / \alpha_{34}$ (искомая величина), $h=\hat{h} / V^{1 / 3}$, $B=g \rho V^{2 / 3} / \alpha_{34}$ (заданные величины). Безразмерная постоянная $B$ - число Бонда.

В безразмерном виде задача примет следующий вид: уравнения Эйлера

$$
\begin{gathered}
\frac{d}{d \xi}\left(\frac{\xi w_{1}^{\prime}(\xi)}{\sqrt{1+\left(w_{1}^{\prime}(\xi)\right)^{2}}}\right)=B \xi w_{1}(\xi)+\mu \xi, \quad \xi_{*}<\xi<\xi_{1} \\
\frac{d}{d \xi}\left(\frac{\xi w_{2}^{\prime}(\xi)}{\sqrt{1+\left(w_{2}^{\prime}(\xi)\right)^{2}}}\right)=B \xi\left(h-w_{2}(\xi)\right)-\mu \xi \\
\xi_{*}<\xi<\xi_{2}
\end{gathered}
$$

условия трансверсальности

$$
\begin{aligned}
& \cos \theta_{1} \equiv \frac{1}{\sqrt{1+\left(w_{1}^{\prime}\left(\xi_{1}\right)\right)^{2}}}=\frac{\alpha_{14}-\alpha_{13}}{\alpha_{34}} \equiv \alpha_{1}, \\
& \cos \theta_{2} \equiv \frac{1}{\sqrt{1+\left(w_{2}^{\prime}\left(\xi_{2}\right)\right)^{2}}}=\frac{\alpha_{24}-\alpha_{23}}{\alpha_{34}} \equiv \alpha_{2} ;
\end{aligned}
$$

условия соприкосновения жидкого моста с дном и крышкой

$$
\begin{aligned}
& w_{1}\left(\xi_{1}\right)=0, \\
& w_{2}\left(\xi_{2}\right)=h ;
\end{aligned}
$$

условие непрерывности профиля моста в шейке

$$
w_{1}\left(\xi_{*}\right)=w_{2}\left(\xi_{*}\right)
$$

условия вертикальности касательной в шейке

$$
w_{1}^{\prime}\left(\xi_{*}\right)=-\infty, w_{2}^{\prime}\left(\xi_{*}\right)=+\infty ;
$$


условие сохранения объема

$$
2 \pi \int_{\xi_{*}}^{\xi_{1}} w_{1}(\xi) \xi d \xi+2 \pi \int_{\xi_{*}}^{\xi_{2}}\left(h-w_{2}(\xi)\right) \xi d \xi+\pi\left(\xi_{*}\right)^{2} h=1 .
$$

\section{Алгоритм решения задачи}

Чтобы упростить зависимость от нижнего предела в интегралах, входящих в (12), и в аналогичных им, введем перенормировку аргумента, искомых функций и параметров:

новая независимая переменная $\eta=\xi / \xi_{*}$;

новые искомые функции $v_{i}(\eta)=w_{i}(\xi) / \xi_{*}, i=1,2$;

новые параметры $H=h / \xi_{*}, b=B\left(\xi_{*}\right)^{2}(b-$ модифицированное число Бонда);

новый множитель Лагранжа $M=\mu \xi_{*}$.

В новых безразмерных переменных уравнения (4) и (5) примут вид

$$
\begin{gathered}
\frac{d}{d \eta}\left(\frac{\eta v_{1}^{\prime}(\eta)}{\sqrt{1+\left(v_{1}^{\prime}(\eta)\right)^{2}}}\right)=b v_{1} \eta+M \eta, \quad 1<\eta<\eta_{1}, \\
\frac{d}{d \eta}\left(\frac{\eta v_{2}^{\prime}(\eta)}{\sqrt{1+\left(v_{2}^{\prime}(\eta)\right)^{2}}}\right)=b\left(H-v_{2}(\eta)\right) \eta-M \eta, \\
1<\eta<\eta_{2} .
\end{gathered}
$$

Интегрируя эти уравнения, получаем

$$
\frac{\eta v_{1}^{\prime}(\eta)}{\sqrt{1+\left(v_{1}^{\prime}(\eta)\right)^{2}}}=b \int_{1}^{\eta} v_{1}(s) s d s+\frac{M\left(\eta^{2}-1\right)}{2}+C_{1},
$$

$\frac{\eta v_{2}^{\prime}(\eta)}{\sqrt{1+\left(v_{2}^{\prime}(\eta)\right)^{2}}}=b \int_{1}^{\eta}\left(H-v_{2}(s)\right) s d s-\frac{M\left(\eta^{2}-1\right)}{2}+C_{2}$.

Переходя в (15) и (16) к пределу при $\eta \rightarrow 1$, находим постоянные $C_{1}$ и $C_{2}: C_{1}=-1, C_{2}=1$. Разделив обе части $(15),(16)$ на $\eta$, приводим эти уравнения к виду

$$
\begin{aligned}
\frac{v_{1}^{\prime}(\eta)}{\sqrt{1+\left(v_{1}^{\prime}(\eta)\right)^{2}}}= & \frac{1}{\eta}\left[b \int_{1}^{\eta} v_{1}(s) s d s+\frac{M\left(\eta^{2}-1\right)}{2}-1\right] \\
\equiv & -\Phi_{1}(\eta), \\
\frac{v_{2}^{\prime}(\eta)}{\sqrt{1+\left(v_{2}^{\prime}(\eta)\right)^{2}}}= & \frac{1}{\eta}\left[b \int_{1}^{\eta}\left(H-v_{2}(s)\right) s d s\right. \\
& \left.-\frac{M\left(\eta^{2}-1\right)}{2}+1\right] \equiv \Phi_{2}(\eta) .
\end{aligned}
$$

Введенные функции $\Phi_{1}$ и $\Phi_{2}$ должны удовлетворять неравенствам $0<\Phi_{1} \leq 1,0<\Phi_{2} \leq 1$. Разрешая (17) и (18) относительно производных, приходим к следующим уравнениям:

$$
\frac{d v_{1}}{d \eta}=-\frac{\Phi_{1}(\eta)}{\sqrt{1-\left(\Phi_{1}(\eta)\right)^{2}}}, \frac{d v_{2}}{d \eta}=\frac{\Phi_{2}(\eta)}{\sqrt{1-\left(\Phi_{2}(\eta)\right)^{2}}}
$$

Из соотношений (17) и (18) следует $\Phi_{1}(1)=\Phi_{2}(1)=1$, а из уравнений (19)

$$
\left.\frac{d v_{1}}{d \eta}\right|_{\eta=1}=-\infty,\left.\quad \frac{d v_{2}}{d \eta}\right|_{\eta=1}=+\infty
$$

т.е. выполнение условий (11). В окрестности точки $\eta=1$ функции $\Phi_{1}$ и $\Phi_{2}$ представляются в виде $\Phi_{i}(\eta)=1+\beta_{i}(\eta-1)+o(\eta-1), i=1,2$, поэтому особенности в правых частях (19) являются интегрируемыми

$$
\begin{gathered}
v_{1}(\eta)=\int_{\eta}^{\eta_{1}} \frac{\Phi_{1}(s) d s}{\sqrt{1-\left(\Phi_{1}(s)\right)^{2}}}, \\
v_{2}(\eta)=H-\int_{\eta}^{\eta_{2}} \frac{\Phi_{2}(s) d s}{\sqrt{1-\left(\Phi_{2}(s)\right)^{2}}},
\end{gathered}
$$

при этом выполнены аналоги условий соприкосновения жидкого моста с дном и крышкой: $v_{1}\left(\eta_{1}\right)=0$, $v_{2}\left(\eta_{2}\right)=H$. Выполняя аналог условия (10), приходим к соотношению

$$
\int_{1}^{\eta_{1}} \frac{\Phi_{1}(s) d s}{\sqrt{1-\left(\Phi_{1}(s)\right)^{2}}}+\int_{1}^{\eta_{2}} \frac{\Phi_{2}(s) d s}{\sqrt{1-\left(\Phi_{2}(s)\right)^{2}}}=H .
$$

Подставляя (17) и (18) в условия трансверсальности

$$
\begin{aligned}
& \left.\frac{v_{1}^{\prime}(\eta)}{\sqrt{1+\left(v_{1}^{\prime}(\eta)\right)^{2}}}\right|_{\eta=\eta_{1}}=-\Phi_{1}\left(\eta_{1}\right)=-\sqrt{1-\alpha_{1}^{2}}, \\
& \left.\frac{v_{2}^{\prime}(\eta)}{\sqrt{1+\left(v_{2}^{\prime}(\eta)\right)^{2}}}\right|_{\eta=\eta_{2}}=\Phi_{2}\left(\eta_{2}\right)=\sqrt{1-\alpha_{2}^{2}},
\end{aligned}
$$

приходим к соотношениям

$$
\begin{aligned}
\frac{1}{2} M \eta_{1}^{2}+ & \sqrt{1-\alpha_{1}^{2}} \eta_{1}-\left[1+\frac{1}{2} M-b \int_{1}^{\eta_{1}} v_{1}(s) s d s\right]=0 \\
\frac{1}{2} M \eta_{2}^{2} & +\sqrt{1-\alpha_{2}^{2}} \eta_{2} \\
- & {\left[1+\frac{1}{2} M+b \int_{1}^{\eta_{2}}\left(H-v_{2}(s)\right) s d s\right]=0 }
\end{aligned}
$$

Считая временно члены, содержащие множитель $b$, известными, решаем (24), (25) как квадратные уравнения 
относительно $\eta_{1}, \eta_{2}$ и получаем соответственно

$$
\begin{aligned}
\eta_{1}^{ \pm}= & \frac{1}{M}\left\{-\sqrt{1-\alpha_{1}^{2}} \pm\left[1-\alpha_{1}^{2}\right.\right. \\
& \left.\left.+2 M\left(1+\frac{1}{2} M-b \int_{1}^{\eta_{1}} v_{1}(s) s d s\right)\right]^{1 / 2}\right\}, \quad(26) \\
\eta_{2}^{ \pm}= & \frac{1}{M}\left\{-\sqrt{1-\alpha_{2}^{2}} \pm\left[1-\alpha_{2}^{2}\right.\right. \\
& \left.\left.+2 M\left(1+\frac{1}{2} M+b \int_{1}^{\eta_{2}}\left(H-v_{2}(s)\right) s d s\right)\right]^{1 / 2}\right\}
\end{aligned}
$$

Перепишем в новых переменных условие сохранения объема (12)

$$
\xi_{*}^{3}\left(\int_{1}^{\eta_{1}} v_{1}(s) s d s+\int_{1}^{\eta_{2}}\left(H-v_{2}(s)\right) s d s+\frac{1}{2} H\right)=\frac{1}{2 \pi}
$$

Интегрируя по частям, приводим это соотношение к виду

$$
\xi_{*}=\left\{\pi\left[\int_{1}^{\eta_{1}} \frac{\Phi_{1}(s) s^{2} d s}{\sqrt{1-\left(\Phi_{1}(s)\right)^{2}}}+\int_{1}^{\eta_{2}} \frac{\Phi_{2}(s) s^{2} d s}{\sqrt{1-\left(\Phi_{2}(s)\right)^{2}}}\right]\right\}^{-1 / 3} .
$$

В результате получаем нелинейную систему четырех уравнений (21), (24), (25), (29) для нахождения четырех параметров $\eta_{1}, \eta_{2}, \xi_{*}, M$.

Последний параметр, который надлежит найти, это модифицированный множитель Лагранжа $M$. Подставляя найденные по формулам (26) и (27) верхние пределы интегрирования $\eta_{1}(M)$ и $\eta_{2}(M)$ в формулу (21), приходим к соотношению

$$
H(M)=\int_{1}^{\eta_{1}(M)} \frac{\Phi_{1}(s) d s}{\sqrt{1-\left(\Phi_{1}(s)\right)^{2}}}+\int_{1}^{\eta_{2}(M)} \frac{\Phi_{2}(s) d s}{\sqrt{1-\left(\Phi_{2}(s)\right)^{2}}} .
$$

Далее можно найти $M$ по заданному значению $H$, обращая функцию (30).

Предлагается для решения задачи использовать метод итераций. А именно:

1. Задаем значение параметра $M$, находим корни квадратных уравнений (24), (25) в предположении, $b=0$, определяем $\xi_{*}, H$ и функции $v_{1}, v_{2}$, определяющие профиль боковой поверхности жидкого моста на первом шаге итерационного процесса.

2. Вычисляем значение $b=B\left(\xi_{*}\right)^{2}$ и выполняем действия описанные выше, но с учетом найденных функций $v_{1}, v_{2}$. Это второй шаг итерационного процесса. Далее делаем третий шаг и так далее до достижения нужной точности вычислений.
При этом проходим весь диапазон допустимых значений параметра $M$ (см. ниже) и строим зависимость безразмерной высоты жидкого моста от этого параметра. Для определения формы боковой поверхности жидкого моста заданной высоты $h-$ находим с помощью построенной зависимости $h(M)$ соответствующие значения параметра $M$, и уже для них выполняем вычисления, описанные выше.

Заметим сразу, что максимальное число таких значений равно четырем (четыре различных профиля моста). При этом, если высота превышает $h_{\max }\left(h_{\max }-\right.$ значение максимальной высоты жидкого моста), то решений нет.

\section{Вертикальный жидкий мост в невесомости (первый шаг итерационного процесса)}

Пусть число Бонда равно нулю. Определяем диапазон допустимых значений параметра $M$. В случае положительных значений параметра $M$ имеем по два вещественных корня разных знаков у каждого из уравнений (24), (25) и выбираем положительные корни $\eta_{1}^{+}, \eta_{2}^{+}$, так как при вычислении интегралов нельзя переходить через точку нуль (см. вид функций $\left.\Phi_{i}(\eta), i=1,2\right)$.

Теперь рассмотрим случай отрицательных значений параметра $M$. Из анализа дискриминантов квадратных уравнений следует, что вещественные корни будут существовать в областях а) $\max \left\{-1+\alpha_{1},-1+\alpha_{2}\right\} \leq M<0$ и б) $M \leq \min \left\{-1-\alpha_{1},-1-\alpha_{2}\right\}$. В области б) корни уравнений (24) и (25) будут меньше единицы и $\xi_{* 0}<0$ (см. (29)). Следовательно, решения, имеющие физический смысл, будут существовать только в области а). Итак, для отрицательных значений параметра $M$ достаточно ограничиться интервалом а). В этом диапазоне каждому значению параметра $M$ будут соответствовать четыре корня уравнений (24), (25), лежащие в области $(+1,+\infty)$, и четыре значения параметра $h$.

Опишем более подробно первый шаг итерационного процесса (первую итерацию). Итак:

1. Задаем значение параметра $M$ из области допустимых значений.

2. Находим положительные корни уравнений (24), (25) при условии $b=0$. Если $M>0$, то их два: $\eta_{1}^{+}, \eta_{2}^{+}$ (см. (26), (27)), а если $M$ принадлежит интервалу $a$ ), то - четыре и появляются соответственно четыре варианта выбора корней для проведения дальнейших вычислений, а именно вариант $(++)$ : корень $\eta_{1}^{+}$уравнения (24) и корень $\eta_{2}^{+}$уравнения $(25)$; вариант $(+-)$ : корни $\eta_{1}^{+}, \eta_{2}^{-}$; вариант $(-+)$и наконец вариант $(--)$.

3. Для каждого из возможных вариантов выбора положительных корней квадратных уравнений (24), (25) вычисляем $\xi_{*}$ по формуле (29) с учетом того, что $\Phi_{1}(\eta)=\Phi_{2}(\eta)=\Phi(\eta)=\left[1-0.5 M\left(\eta^{2}-1\right)\right] / \eta$.

4. Находим $H$ по формуле $(21)$ и функции $v_{1}(\eta), v_{2}(\eta)$ по формулам (20). 


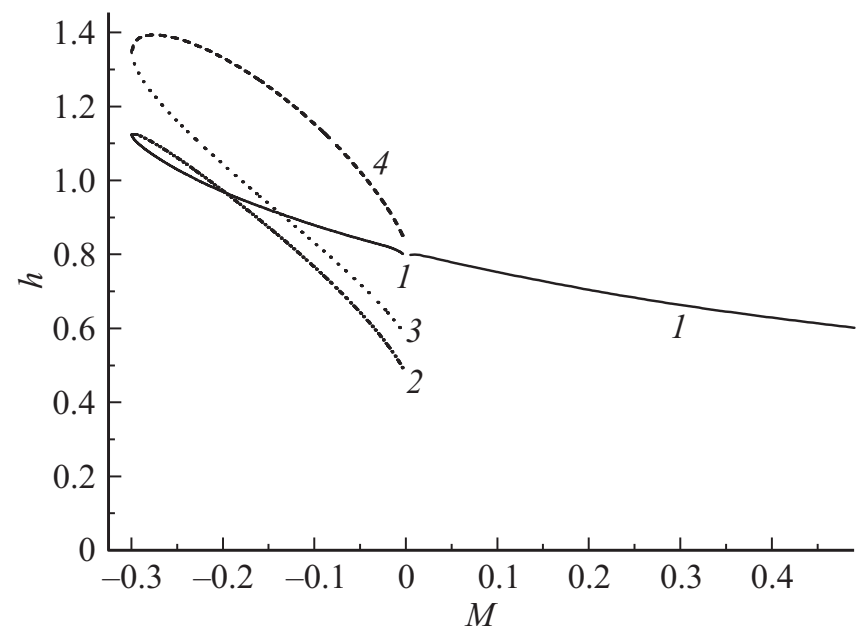

Рис. 2. Зависимость высоты жидкого моста $h$ от параметра $M$. Случай $\alpha_{1}=0.5, \alpha_{2}=0.7$. График 1 соответствует варианту $(++)$ выбора корней уравнений $(24),(25) ; 2$ - варианту $(+-)$, 3 - варианту $(-+), 4$ - варианту (--).

5. Возвращаемся к исходным безразмерным переменным и находим $\xi_{1}, \xi_{2}, h$, а затем функции $w_{1}(\xi)=v_{1}\left(\xi / \xi_{*}\right) \xi_{*}, w_{2}(\xi)=v_{2}\left(\xi / \xi_{*}\right) \xi_{*}$, описывающие в первом приближении профиль жидкого моста в каждом из вариантов.

Далее строим график зависимости величины безразмерной высоты жидкого моста от параметра $M$. Для случая $\alpha_{1}=0.5, \alpha_{2}=0.7$ она приведена на рис. 2 .

Видим, что в области положительных значений параметра $M$ при его возрастании от нуля до $+\infty$ высота жидкого моста $h$ убывает монотонно от своего значения при $M=0$ до нуля. В области отрицательных значений параметра существуют четыре ветви, соответствующие вариантам выбора корней квадратных уравнений (ветви $(++),(+-),(-+),(--))$. Кроме того, из этого графика видно, что достаточно малым значениям $h$ будет соответствовать одно значение параметра $M$, а значит, только один профиль жидкого моста.

Итак, одному фиксированному значению $h$ на первом шаге итерационного процесса могут соответствовать четыре различных профиля капли (максимальное число ветвей). Эту ситуацию иллюстрирует рис. 3. На нем изображены четыре различных профиля жидкого моста, соответствующие одному значению безразмерной высоты $h=1$, причем рассматривается симметричный случай $\alpha_{1}=\alpha_{2}=0.5$.

\section{Вертикальный жидкий мост (второй шаг итерационного процесса)}

Перейдем к описанию второго шага итерационного процесса (второй итерации). Здесь уже действие силы тяжести на вертикальный жидкий мост учитывается.
Второй шаг итерационного процесса предполагает выполнение следующих действий:

1. При том же заданном значение параметра $M$ находим положительные корни уравнений (24), (25), используя соотношения (26) и (27). Величины $\xi_{*}, \eta_{1}, \eta_{2}$, а также функции $v_{1}(\eta), v_{2}(\eta)$, берем из результатов расчета первого приближения.

2. Для каждого из вариантов выбора корней вычисляем новое значение $\xi_{*}$ по формуле (29) (функции $\Phi_{1}(\eta), \Phi_{2}(\eta)$ определяем по формулам $\left.(17),(18)\right)$.

3. Находим $H$ по формуле $(21)$ и функции $v_{1}(\eta), v_{2}(\eta)$ по формулам (20).

4. Возвращаемся к исходным безразмерным переменным и находим $\xi_{1}, \xi_{2}, h$, а затем функции $w_{1}(\xi)=$ $=v_{1}\left(\xi / \xi_{*}\right) \xi_{*}, w_{2}(\xi)=v_{2}\left(\xi / \xi_{*}\right) \xi_{*}$, описывающие во втором приближении профиль жидкого моста в каждом из вариантов.

Следующие итерации могут быть выполнены по тому же алгоритму, что и вторая итерация.

В качестве примера проведем расчет профиля жидкого моста с учетом одной и двух итераций. Результаты расчета в варианте $(++)$ для $\alpha_{1}=0.5, \alpha_{2}=0.7$, $M=-0.273$ приведены на рис. 4. Видно, что учет второй итерации при достаточно малых значениях параметра $b$ незначительно меняет решение.

\section{Асимптотика решения в окрестности точки $M=0$ и поиск максимально возможной высоты жидкого моста}

Построим асимптотику решения, полученного в результате одного шага итерационного процесса (случай $b=0)$ в окрестности точки $M=0$. В случае сильной смачиваемости эта точка будет левой границей области допустимых значений параметра $M$. В каждом из вариантов выбора корней квадратных уравнений (24), (25) асимптотика своя.

Рассмотрим вариант $(++)$ и предположим, что параметр $M \rightarrow 0$. В этом случае можно аппроксимировать вспомогательную функцию $\Phi$ следующим образом: $\Phi(\eta)=1 / \eta$. Выполнив интегрирование в (29), (21) с учетом

$$
\eta_{1} \sim\left(1-\alpha_{1}^{2}\right)^{-1 / 2}, \quad \eta_{2} \sim\left(1-\alpha_{2}^{2}\right)^{-1 / 2},
$$

получим приближенно

$$
\begin{gathered}
\xi_{*} \sim\left(\frac{2}{\pi}\right)^{1 / 3}\left\{\frac{\alpha_{1}}{1-\alpha_{1}^{2}}+\frac{\alpha_{2}}{1-\alpha_{2}^{2}}\right. \\
\left.+\frac{1}{2} \ln \left(\frac{\left(1+\alpha_{1}\right)\left(1+\alpha_{2}\right)}{\left(1-\alpha_{1}\right)\left(1-\alpha_{2}\right)}\right)\right\}^{-1 / 3}, \\
H \sim \frac{1}{2} \ln \left(\frac{\left(1+\alpha_{1}\right)\left(1+\alpha_{2}\right)}{\left(1-\alpha_{1}\right)\left(1-\alpha_{2}\right)}\right) .
\end{gathered}
$$



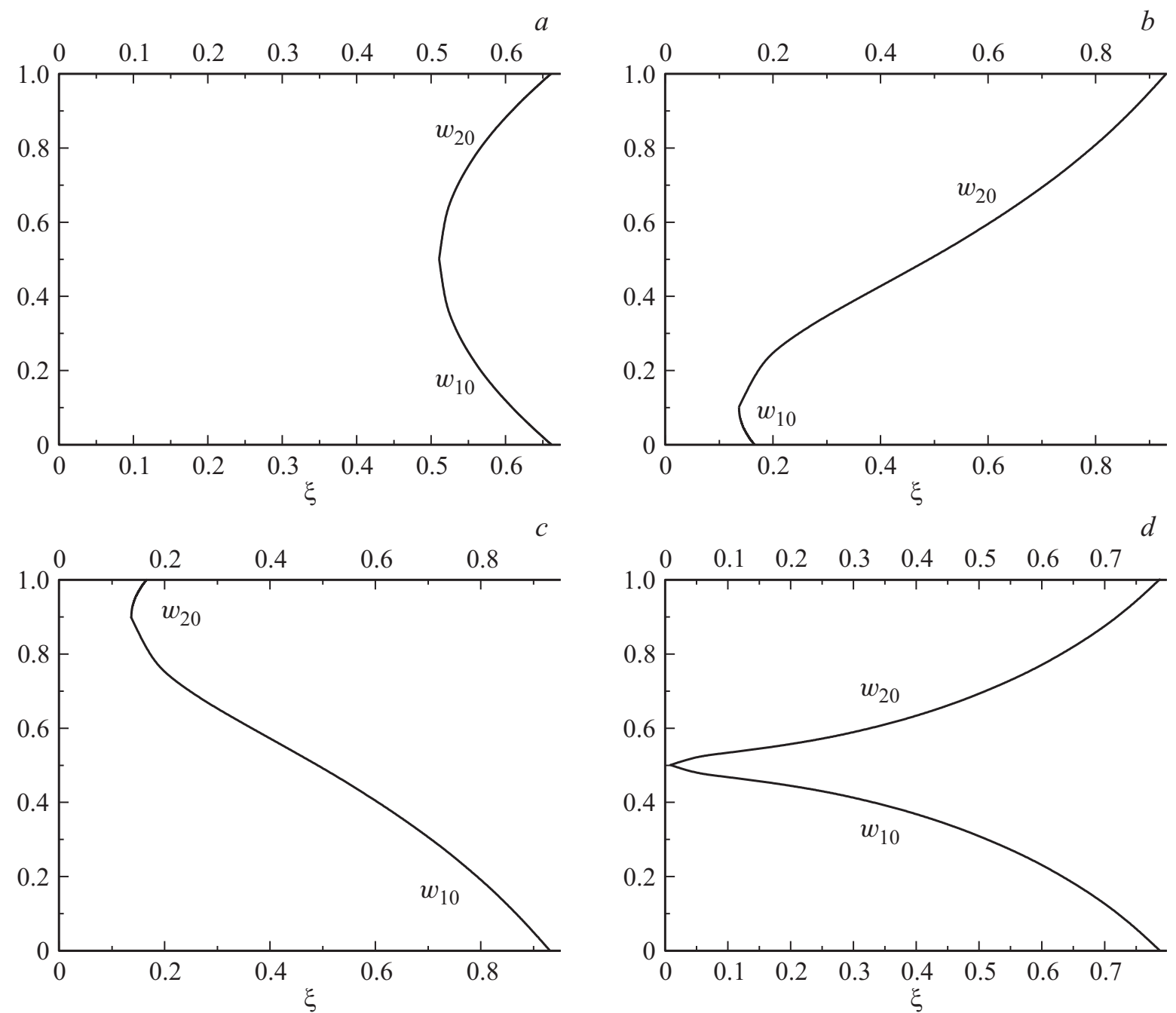

Рис. 3. Четыре различных профиля жидкого моста, соответствующие одному значению его высоты $h=1.0$. Случай $\alpha_{1}=\alpha_{2}=0.5$. $a-$ вариант $(++), M=-0.36 ; b-(+-), M=-0.216 ; c-(-+), \quad M=-0.216 ; d-(--), \quad M=-0.019$.

Кроме того, из (20) найдем приближенные выражения, описывающие форму боковой поверхности жидкого моста,

$$
\begin{gathered}
v_{1}(\eta) \sim \ln \left(\left(1+\alpha_{1}\right)^{1 / 2}\left(1-\alpha_{1}\right)^{-1 / 2}\left[\eta+\left(\eta^{2}-1\right)^{1 / 2}\right]^{-1}\right), \\
v_{2}(\eta) \sim \ln \left(\left(1+\alpha_{1}\right)^{1 / 2}\left(1-\alpha_{1}\right)^{-1 / 2}\left[\eta+\left(\eta^{2}-1\right)^{1 / 2}\right]\right) .
\end{gathered}
$$

Варианты $(+-),(-+),(--)$ реализуются только для отрицательных значений параметра $M$ и будем рассматривать случай $M \rightarrow-0$. Здесь необходима более точная аппроксимация подынтегральных выражений в интегралах (21), (29), а именно

$$
\frac{\Phi(\eta)}{\sqrt{1-(\Phi(\eta))^{2}}} \sim \frac{1-0.5 M\left(\eta^{2}-1\right)}{\sqrt{\eta^{2}-1}} .
$$

Тогда для варианта $(+-)$ получим

$$
\eta_{1} \sim\left(1-\alpha_{1}^{2}\right)^{-1 / 2}, \quad \eta_{2} \sim \frac{-2}{M}\left(1-\alpha_{2}^{2}\right)^{1 / 2},
$$

$$
\begin{gathered}
\xi_{*} \sim-M(2 \pi)^{-1 / 3}\left(1-\alpha_{2}^{2}\right)^{-2 / 3}, \\
\xi_{1} \sim-M(2 \pi)^{-1 / 3}\left(1-\alpha_{1}^{2}\right)^{-1 / 2}\left(1-\alpha_{2}^{2}\right)^{-2 / 3}, \\
\xi_{2} \sim(2)^{2 / 3}(\pi)^{-1 / 3}\left(1-\alpha_{2}^{2}\right)^{-1 / 6}, \quad H \sim \frac{-\left(1-\alpha_{2}^{2}\right)}{M}, \\
h \sim(2 \pi)^{-1 / 3}\left(1-\alpha_{2}^{2}\right)^{1 / 3} .
\end{gathered}
$$

Для варианта $(-+)(M \rightarrow-0)$ :

$$
\begin{gathered}
\eta_{1} \sim \frac{-2}{M}\left(1-\alpha_{1}^{2}\right)^{1 / 2}, \quad \eta_{2} \sim\left(1-\alpha_{2}^{2}\right)^{-1 / 2}, \\
\xi_{*} \sim-M(2 \pi)^{-1 / 3}\left(1-\alpha_{1}^{2}\right)^{-2 / 3}, \\
\xi_{1} \sim(2)^{2 / 3}(\pi)^{-1 / 3}\left(1-\alpha_{1}^{2}\right)^{-1 / 6}, \\
\xi_{2} \sim-M(2 \pi)^{-1 / 3}\left(1-\alpha_{1}^{2}\right)^{-2 / 3}\left(1-\alpha_{2}^{2}\right)^{-1 / 2}, \\
H \sim \frac{-\left(1-\alpha_{1}^{2}\right)}{M}, \\
h \sim(2 \pi)^{-1 / 3}\left(1-\alpha_{1}^{2}\right)^{1 / 3} .
\end{gathered}
$$




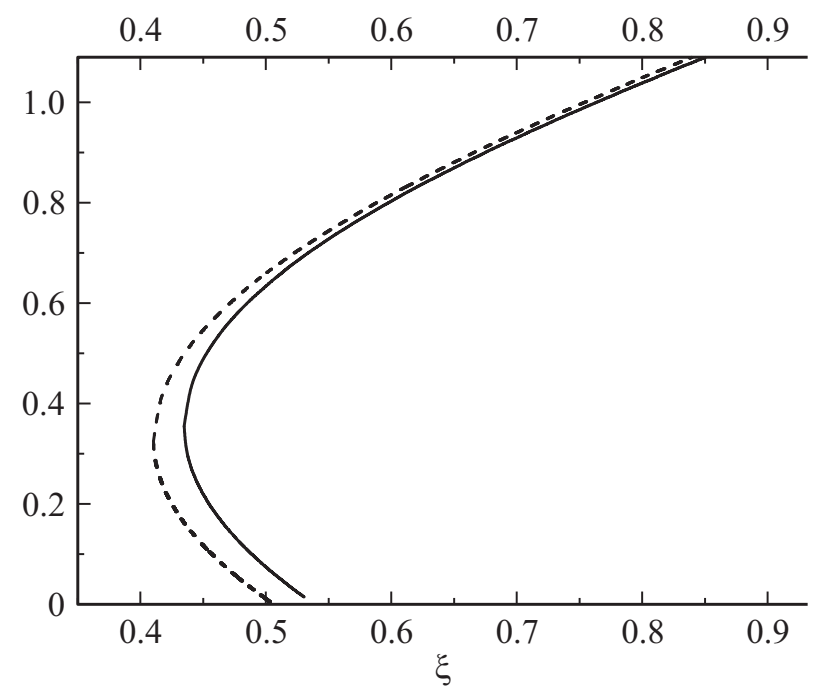

Pис. 4. Профили жидкого моста после первого шага итерационного процесса (сплошная линия) и после второго шага (штриховая линия). Случай $\alpha_{1}=0.5, \alpha_{2}=0.7, M=-0.273$, $B=0.224, b=0.042$. Вариант $(++)$.

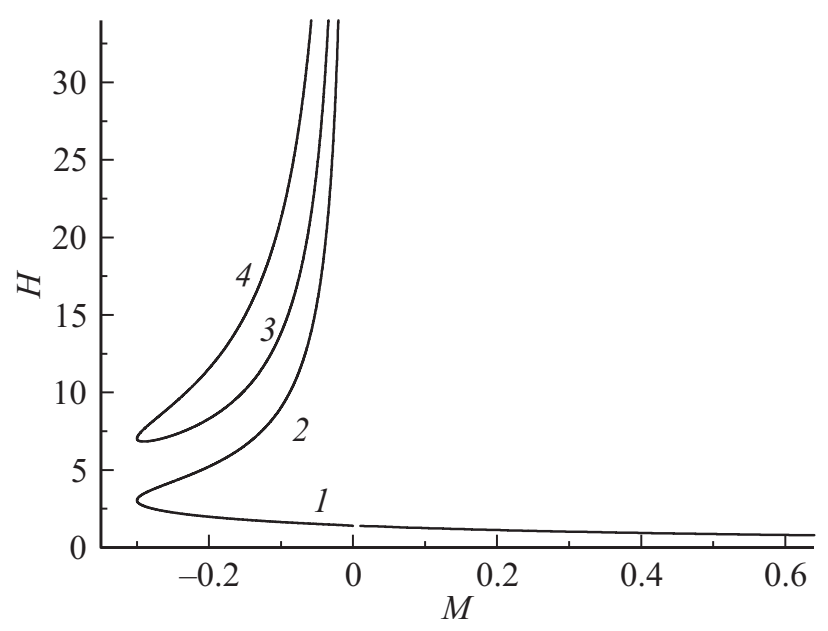

Рис. 5. Зависимость величины $H$ от параметра $M$. Случай $\alpha_{1}=0.5, \alpha_{2}=0.7$. График 1 соответствует варианту $(++)$ выбора корней уравнений (24), (25); $2-(+-) ; 3-$ варианту $(-+)$, график $4-$ варианту $(--)$.

Для варианта $(--)(M \rightarrow-0)$ :

$$
\begin{gathered}
\eta_{1} \sim \frac{-2}{M}\left(1-\alpha_{1}^{2}\right)^{1 / 2}, \quad \eta_{2} \sim \frac{-2}{M}\left(1-\alpha_{2}^{2}\right)^{1 / 2}, \\
\xi_{*} \sim-M\left(2 \pi\left[\left(1-\alpha_{1}^{2}\right)^{2}+\left(1-\alpha_{2}^{2}\right)^{2}\right]\right)^{-1 / 3}, \\
\xi_{1} \sim(2)^{2 / 3}\left(1-\alpha_{1}^{2}\right)^{1 / 2}\left(\pi\left[\left(1-\alpha_{1}^{2}\right)^{2}+\left(1-\alpha_{2}^{2}\right)^{2}\right]\right)^{-1 / 3}, \\
\xi_{2} \sim(2)^{2 / 3}\left(1-\alpha_{2}^{2}\right)^{1 / 2}\left(\pi\left[\left(1-\alpha_{1}^{2}\right)^{2}+\left(1-\alpha_{2}^{2}\right)^{2}\right]\right)^{-1 / 3},
\end{gathered}
$$

$$
\begin{gathered}
H \sim \frac{-\left(2-\alpha_{1}^{2}-\alpha_{2}^{2}\right)}{M}, \\
h \sim(2 \pi)^{-1 / 3}\left(2-\alpha_{1}^{2}-\alpha_{2}^{2}\right)\left[\left(1-\alpha_{1}^{2}\right)^{2}+\left(1-\alpha_{2}^{2}\right)^{2}\right]^{-1 / 3} .
\end{gathered}
$$

На рис. 5 приведена зависимость величины $H$ от параметра $M$ при $\alpha_{1}=0.5, \alpha_{2}=0.7$ для четырех вариантов выбора корней квадратных уравнений (ветви $(++),(+-),(-+),(--))$. Видно, что поведение кривых в окрестности точки $M=0$ соответствует приведенной выше асимптотике решения.

Рассмотрим теперь случай сильной смачиваемости (случай малого угла смачивания) хотя бы на одной из твердых плоскостей. Пусть, например, $\theta_{1} \rightarrow 0$ и, следовательно, $\alpha_{1} \rightarrow 1$. Тогда очевидно область а) стягивается в точку и левая граница области допустимых значений параметра $M$ есть точка $M=0$ и реализуется вариант $(++)$. Соотношения (31) в этом случае (при $\left.M=0, \alpha_{1} \rightarrow 1\right)$ дают

$$
\begin{gathered}
\xi_{*} \sim(2)^{2 / 3}(\pi)^{-1 / 3}\left(1-\alpha_{1}\right)^{1 / 3}, \quad H \sim-0.5 \ln \left(1-\alpha_{1}\right), \\
h \sim-(2 \pi)^{-1 / 3}\left(1-\alpha_{1}\right)^{1 / 3} \ln \left(1-\alpha_{1}\right), \\
\xi_{1} \sim(2)^{1 / 6}(\pi)^{-1 / 3}\left(1-\alpha_{1}\right)^{-1 / 6} \\
\xi_{2} \sim(2)^{2 / 3}(\pi)^{-1 / 3}\left(1-\alpha_{2}^{2}\right)^{-1 / 2}\left(1-\alpha_{1}\right)^{1 / 3}
\end{gathered}
$$

Видно, что $\xi_{*} \rightarrow 0, \xi_{1} \rightarrow+\infty, \xi_{2} \rightarrow 0, \quad h \rightarrow 0 \quad$ при $\alpha_{1} \rightarrow 1$.

Перейдем к обсуждению вопроса о максимальном значении безразмерной высоты жидкого моста $h$ между двумя твердыми плоскостями. Из общих соображений ясно, что такое значение может достигаться только при углах смачивания $\theta_{1}, \theta_{2}$, стремящихся к $\pi / 2$. Как следует из приведенной выше зависимости величины $h$ от параметра $M$ при условии $b=0$ (рис. 2), максимальное значение высоты моста достигается в варианте $(--)$. Заметим, что такое же расположение ветвей будет иметь место и при $\alpha_{1}=\alpha_{2} \rightarrow 0$. Анализируя результаты численных расчетов, проведенных для варианта $(--)$ при $b=0, \alpha_{1}=\alpha_{2}=0, M \rightarrow-1$, находим максимальное значение $h$ и оно равно $h_{\max } \simeq 2.325$.

Интегралы в формулах (21) и (29) выражаются через эллиптические интегралы первого и второго рода

$$
\begin{aligned}
\int_{1}^{\eta} \frac{\Phi(s) d s}{\sqrt{1-(\Phi(s))^{2}}}= & F\left(\frac{\pi}{2}, k\right)-F(\tau(\eta), k) \\
& +\frac{1+\delta}{1-\delta}\left(E\left(\frac{\pi}{2}, k\right)-E(\tau(\eta), k)\right),
\end{aligned}
$$




$$
\begin{gathered}
\int_{1}^{\eta} \frac{\Phi(s) s^{2} d s}{\sqrt{1-(\Phi(s))^{2}}}=-\frac{1+\delta}{3(1-\delta)}\left\{F\left(\frac{\pi}{2}, k\right)-F(\tau(\eta), k)\right. \\
-\frac{7+\delta^{2}}{(1-\delta)^{2}}\left[E\left(\frac{\pi}{2}, k\right)-E(\tau(\eta), k)\right] \\
\left.+\eta\left(\eta^{2}-1\right)^{1 / 2}\left(1-\frac{(1-\delta)^{2} \eta^{2}}{(1+\delta)^{2}}\right)^{1 / 2}\right\}
\end{gathered}
$$

где

$$
\delta=M+1, \tau(\eta)=\arcsin \left(\frac{1-(1-\delta)^{2}(1+\delta)^{-2} \eta^{2}}{k}\right),
$$

$F(\varphi, k), E(\varphi, k)$ - эллиптические интегралы первого и второго рода в форме Лежандра, $k$ - их модуль, в нашем случае $k=2 \sqrt{\delta} /(1+\delta)[10]$.

Используя эти представления, можно найти формулу для найденной численно максимальной высоты жидкого моста, а именно

$$
h_{\max }=(2)^{2 / 3}(\pi)^{1 / 3} .
$$

\section{Заключение}

В работе дана вариационная постановка задачи о вертикальном жидком мосте между двумя параллельными твердыми плоскостями с учетом силы тяжести в осесимметричном случае (в цилиндрической системе координат). Построен итерационный процесс нахождения ее приближенного решения в предположении малости числа Бонда. Показано, что учет второго шага итерационного процесса при достаточно малых значениях модифицированного числа Бонда не вносит существенных изменений в форму профиля жидкого моста.

Обнаружено отсутствие единственности решения: при фиксированной высоте жидкого моста (расстоянии между дном и крышкой) может существовать несколько решений (несколько различных профилей боковой поверхности капли), причем их максимальное число равно четырем. При значениях высоты жидкого моста, превышающих значение $h=h_{\max }$, решений не существует. При значениях высоты, меньших некоторого значения, имеем только один профиль поверхности.

Для случая сильной смачиваемости построена асимптотика поведения основных параметров жидкого моста в окрестности левой границы области допустимых значений параметра $M$.

Найдена максимальная высота жидкого вертикального моста на первом шаге итерационного процесса.

\section{Список литературы}

[1] Финн Р. Равновесные капиллярные поверхности. Математическая теория. М.: Мир, 1989. 312 с.

[2] Галактионов Е.В., Галактионова Н.Е., Тропn Э.А. // ЖТФ. 2016. Т. 86. Вып. 12. С. 17-24.
[3] Антонов П.И., Затуловский Л.М., Костыгов А.С. и др. Получение профилированных монокристаллов и изделий способом Степанова / Под ред. В.Р. Регеля, С.П. Никанорова. Л.: Наука, 1981. 280 с.

[4] Гегузин Я.Е. Капля. М.: Наука, 1973. 160 с.

[5] Галактионов Е.В., Демидович Н.Е., Тропn Э.А. В сб.: Вопросы математической физики и прикладной математики / Под ред. Е.В. Галактионова. СПб.: ФТИ им. А.Ф. Иоффе, 2010. $232 \mathrm{c}$.

[6] Fortes M.A.// J. Colloid Interface Sci. 1982. Vol. 88. N 2. P. 338.

[7] Fel L.G., Rubinstein B.Y. // Z. Angew. Math. 2016. Vol. 66. P. 3447.

[8] Vogel T. // Pac. J. Math. 2006. Vol. 224. N 2. P. 367.

[9] Haynes M., O'Brien S.B.G., Benilov E.S. // Phys. Fluids. 2016. Vol. 28. P. 042107.

[10] Фихтенгольи Г.М. Курс дифференциального и интегрального исчисления. Т. 2. М.: ГИФ-МЛ, 1959. 807 с. 